\title{
Taking decisions in the diagnostic intelligent systems on the basis information from an artificial neural network
}

\author{
Stanisław Duer, ${ }^{1,}$, Serghei Scaticailov ${ }^{2}$, Jacek Paś $^{3}$, Radosław Duer ${ }^{4}$ and Dariusz \\ Bernatowicz ${ }^{4}$ \\ ${ }^{1}$ Koszalin University of Technology, Department of Mechanical Engineering, Poland \\ ${ }^{2}$ Technical University of Moldova, Department of Manufacturing Engineering, Republic of Moldova \\ ${ }^{3}$ Military University of Technology, Warsaw, Department of Electronic, Poland \\ ${ }^{4}$ Koszalin University of Technology, Department of Electronic and Computer Science, Poland
}

\begin{abstract}
This paper presents a method to control an operation process of a complex technical object, with the use of trivalent diagnostic information. Also, a general diagram of the complex technical object was presented, and its internal structure was described. A diagnostic analysis was conducted, as a result of which sets of the functional elements of the object and its diagnostic signals were determined. Also, the methodology of the diagnostic examination of the technical system was presented. The result was a functional and diagnostic model, which constituted the basis for initial diagnostic information, which is provided by the sets of information concerning the elements of the basic modules and their output signals. The theoretical results obtained in the present study were verified in practice on the example of a complex and reparable technical object. It belongs to the group of technical equipment for which a short time of shutdowns is required (an ineffective use of the object).
\end{abstract}

\section{Introduction}

Technical diagnostics facility provides the user with diagnostic information for the organization of the use. A particular example is the information expressed in trivalent logic. Diagnosing technical objects in trivalent logic identifies the object states: fitness - $\{2\}$, the state of unfitness - $\{0\}$, and the incomplete state of fitness - $\{1\}[1,2-6]$. The resulting diagnostic information about the object with such a diagnosis, due to the recognition of the suitability of the incomplete state of the inside of the object of knowledge is the basis for deciding maintenance or use organizing strategies of constructing a new direction in the handling of technical objects called the handling of the prior [5-7, 9, 10, 12]. In this situation, when these two areas of expertise: theory and the theory of diagnostic use of technical facilities, cooperate with each other in the organization of the use of a technical object, you can no longer talk about the operating system.

\footnotetext{
*Corresponding author: stanislaw.duer@tu.koszalin.pl
} 
The knowledge of the operation process of a technical object through the determination of the times of the occurrence of repairs (shutdown) and operation will make it possible to control this process (Figure 1).

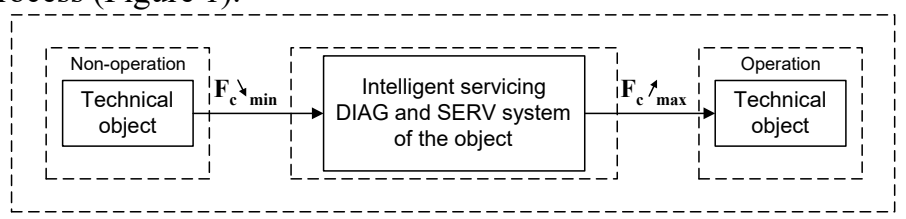

Fig. 1. Diagram of an intelligent system for regulation agreement of the quality of the use of the object $\left(\mathrm{F}_{\mathrm{C}}\right)$.

Where: $F_{C \min }, \mathrm{F}_{\mathrm{Cmax}}-\min$. and max. value of function of the use of the object, DIAG - is a powerful computer software, a hybrid, a combination of neural network with the module diagnostic inference. DIAG is designed to diagnose analog-class technical objects, SERV Expert software is a computer program designed to assist in the process of determining the knowledge base maintenance of the technical object.

The application of an automatic adjustment and control system (Figure 1) of the level of the functional properties of a technical object constitutes an appropriate approach to the issue of a qualitative control of the use of a technical object in the process of operation. The purpose of this adjustment system is to maintain in an automatic and continuous manner the functional properties on the required level (which guarantees a qualitative use of the object). The automatic diagnostic system (Figure 1) utilizes not only a measurement A/D converter card with appropriate signal interfaces but also some computer tool used for proper signal registration as well as for acquiring and processing data registered $[7,8,11$, 12].

The purpose of such a process is to build a diagnostic knowledge base basing upon an analysis of both the object and the results of the measurement stored.

\section{The intelligent diagnostic system of the technical object}

The object's state is determined on the basis of an examination of the set of output (diagnostic) signals $\left\{X\left(e_{i, j}\right)\right\}(\mathrm{Tab} 1)[4,5,7,9]$. The set of its functional elements $\left\{e_{i, j}\right\}$ determined during a diagnostic study of the object constitutes the basis for the list included in the table of a set of diagnostic signals (Table 1).

Table 1. Table of object's input diagnostic signals.

\begin{tabular}{|c|c|c|c|c|c|c|}
\hline Object & \multirow{2}{*}{$\begin{array}{c}\text { Level of } \\
\text { object } \mathrm{E}_{\mathrm{i}}\end{array}$} & \multicolumn{5}{|c|}{ Vector of initial diagnostic signals $\left\{X\left(e_{i, j}\right)\right\}$} \\
\cline { 3 - 7 } & $X\left(\mathrm{e}_{1,1}\right)$ & $\ldots$ & $X\left(\mathrm{e}_{\mathrm{i}, \mathrm{j}}\right)$ & $\ldots$ & $X\left(e_{i, J}\right)$ \\
\hline \multirow{4}{*}{$\mathrm{E}$} & $E_{I}$ & $X\left(\mathrm{e}_{1,1}\right)$ & $\ldots$ & $X\left(e_{1, j}\right)$ & $\ldots$ & $X\left(e_{1, J}\right)$ \\
\cline { 2 - 7 } & $\vdots$ & $\vdots$ & $\ldots$ & $\vdots$ & $\ldots$ & $\vdots$ \\
\cline { 2 - 7 } & $E_{i}$ & $X\left(\mathrm{e}_{\mathrm{i}, 1}\right)$ & $\ldots$ & $X\left(e_{i, j}\right)$ & $\ldots$ & $X\left(e_{i, J}\right)$ \\
\cline { 2 - 7 } & $\vdots$ & $\vdots$ & $\ldots$ & $\vdots$ & $\ldots$ & $\vdots$ \\
\cline { 2 - 7 } & $E_{I}$ & $X\left(\mathrm{e}_{\mathrm{I}, 1}\right)$ & $\ldots$ & $X\left(e_{I, j}\right)$ & $\ldots$ & $X\left(e_{I, J}\right)$ \\
\hline
\end{tabular}

Where: $X\left(e_{i, j}\right)$ - diagnostic signal of $j^{\text {th }}$ element in $i^{\text {th }}$ assembly, $E_{i}-i^{\text {th }}$ functional assembly of the object, $e_{j}-j^{\text {th }}$ subassembly in $i^{\text {th }}$ assembly of the object.

The realization of the task of a comparison of the image of the diagnostic signal with the image of its standard (nominal) signal is the purpose of the neural network in that is used in the diagnostic system [4, 7]. For this purpose, it is useful to present the images of the diagnostic signals compared in a vector form. The analytical form of the equation which describes the diagnostic process of technical objects (Figure 1) performed with a comparison method of signals with their model, was presented in the form of the following dependence: 


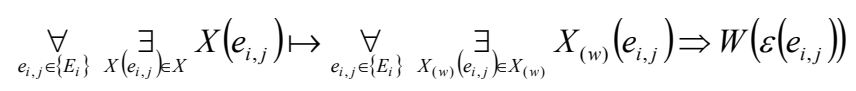

Where: $W\left(\varepsilon\left(e_{i, j}\right)\right)$ - value of state assessment logics for $j^{\text {th }}$ element within $i^{\text {th }}$ module, $X\left(e_{i, j}\right)$ - diagnostic signal in $j^{\text {th }}$ element of $i^{\text {th }}$ of the object, $X_{(w)}\left(e_{i, j}\right)$ - model signal for $X\left(e_{i, j}\right)$ signal, $E_{i}-i^{\text {th }}$ functional assembly of the object.

The effect of such an activity (comparison) of signals is a decision, which is worked out by the network, as to which of the three distinguished states $\{2,1,0\}$ a given element of the object which worked out this signal, is to be qualified to.

In the literature which describes neural networks Minkowski measure is used in network methods based on the rule of similarity for an analysis of the metrics of vectors of signals [4-7]. It is presented in the form of the following dependence:

$$
D_{M}\left(X_{i}, X_{(w) i}, \alpha\right)=\left(\sum_{i=1}^{N}\left|X_{i}-X_{(w)_{i}}\right|^{\alpha}\right)^{1 / \alpha}
$$

Where: $D_{M}\left(X_{i}, X_{(w) i} \alpha\right)$, - standard deviation of the vector the signal metric, $(\alpha=2)$.

The network whose diagram is presented in (Figure 2) consists of three layers: $F_{1}-$ input layer, $F_{2}$ - hidden layer and $F_{3}$ - output layer. The neural cells of the network process the diagnostic information according to the algorithm (Figure 2).

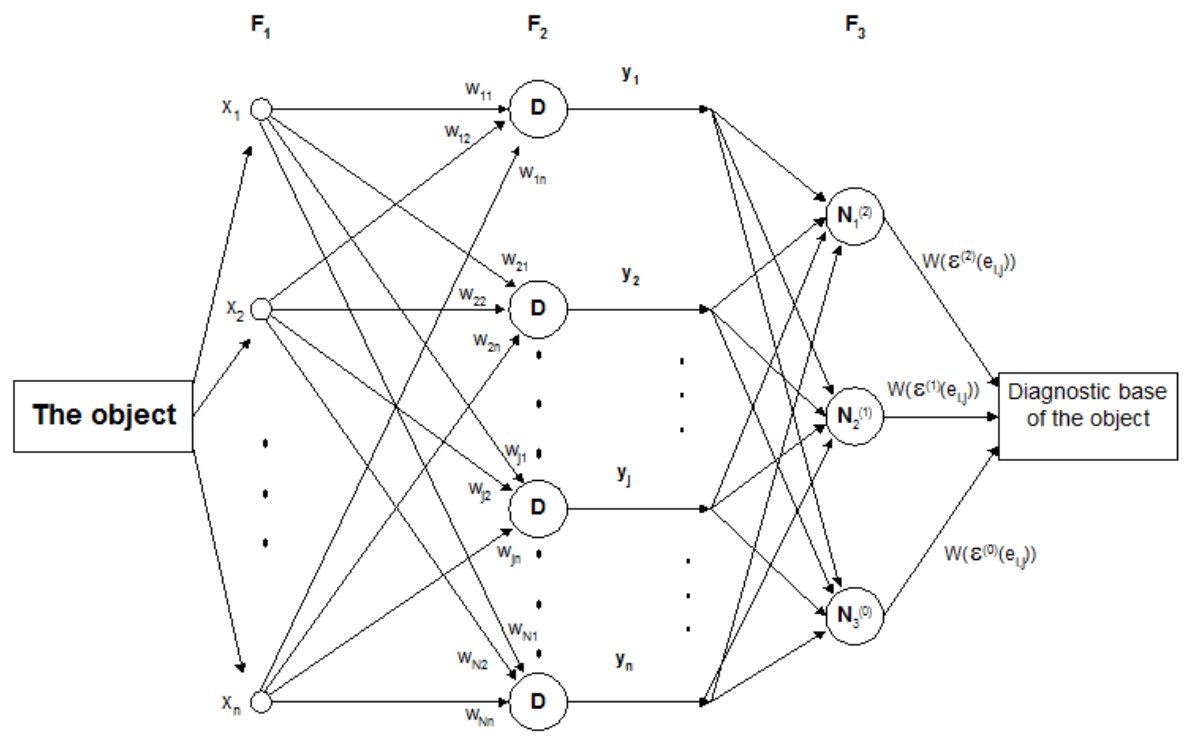

Fig. 2. Structure of an artificial neural network in the DIAG system.

The results were obtained from the diagnosis of the object examined according to the diagram presented in (Figure 2), which are calculated as a result of the realization of dependences (1 and 2), which are set up in the form of the table of states (Table 2). 
Table 2. Table of the states of the object's elements.

\begin{tabular}{|c|c|c|c|c|c|}
\hline $\begin{array}{c}\text { Number } \\
\text { of the } \\
\text { assembly }\end{array}$ & \multicolumn{6}{|c|}{$\begin{array}{c}\text { ector of the states of basic elements in } \\
\text { the structure of the object }\left\{\mathrm{e}_{\mathrm{i}, \mathrm{j}}\right\}\end{array}$} \\
\hline & $\varepsilon\left(e_{1,1}\right)$ & $\ldots$ & $\varepsilon\left(e_{i, j}\right)$ & $\ldots$ & $\varepsilon\left(e_{i, J}\right)$ \\
\hline$E_{I}$ & $W\left(\varepsilon\left(e_{1,1}\right)\right)$ & $\ldots$ & $W\left(\varepsilon\left(e_{1, j}\right)\right)$ & $\ldots$ & $W\left(\varepsilon\left(e_{1, J}\right)\right)$ \\
\hline$\vdots$ & $\vdots$ & $\ldots$ & $\vdots$ & $\ldots$ & $\vdots$ \\
\hline$E_{i}$ & $W\left(\varepsilon\left(e_{\mathrm{i}, 1}\right)\right)$ & $\ldots$ & $W\left(\varepsilon\left(e_{i, j}\right)\right)$ & $\ldots$ & $\varnothing$ \\
\hline$\vdots$ & $\vdots$ & $\ldots$ & $\vdots$ & $\ldots$ & $\vdots$ \\
\hline$E_{I}$ & $W\left(\varepsilon\left(e_{I, 1}\right)\right)$ & $\ldots$ & $W\left(\varepsilon\left(e_{I, j}\right)\right)$ & $\ldots$ & $W\left(\varepsilon\left(e_{I, J}\right)\right)$ \\
\hline
\end{tabular}

Where: $W\left(\varepsilon\left(e_{i, j}\right)\right)$ - is the value of the state of $j^{\text {th }}$ element in $i^{\text {th }}$ unit (from the set of the accepted trivalent logic of the assessment of states $-\{2,1,0\}), \varnothing$ is the element which complements the dimension of the table.

\section{Taking decisions in the diagnostic intelligent systems on the basis information from an artificial neural network}

The neural network of the diagnostic system (Figure 3) identifies - classifies the states of the set of distinguished basic elements in the structure of the object $\left\{e_{i, j}\right\}$. The results of the diagnosis of the object are presented in the final form in (Table 1).

The current state of the functional units of the object $\left\{E_{i}\right\}$ and the state of the object under examination $\{\mathrm{O}\}$ is determined on two stages by the inference module in the diagnostic system. On the first stage, the diagnostic module determines the state of $\left(i^{\text {th }}\right)$ units of the object. For this purpose, the states of $\left(j^{\text {th }}\right)$ functional elements are analyzed in a given $\left(i^{\text {th }}\right)$ unit of the object. The inference rule to identify the state of $\left(i^{\text {th }}\right)$ functional unit of the object takes the form of the following dependence:

$$
W\left(\varepsilon\left(E_{i}\right)\right)=\min _{1 \leq j \leq J}\left\{W\left(\varepsilon\left(e_{j}\right)\right)\right\}
$$

Where: $W\left(\varepsilon\left(E_{i}\right)\right)$ - is the value of the state of $i^{\text {th }}$ functional unit of the object, $W\left(\varepsilon\left(e_{i, j}\right)\right)$ - is the value of the state of $j^{\text {th }}$ element in $i^{\text {th }}$ unit (from the set of the accepted trivalent logic of the assessment of states $-\{2,1,0\}$ ). The idea of the identification of the states of units presented in the form of dependence (3) is graphically depicted in (Figure 3).

It results from the analysis in (Figure 3 ) that if at least one of the states of the elements contained in the structure of a given $\left(i^{\text {th }}\right)$ unit is in the state $\left(W\left(\varepsilon\left(e_{j}\right)\right)\right)$ with the lowest valence marked from set $-\{2,1,0\}$, the state of a given unit, which is interpreted with the inference rule (dependence 3 ), is such as the lowest state of this $\left(j^{\text {th }}\right)$ element.

The state of the object of diagnosis is determined on the second stage of the work of the diagnostic module. The determined set of states of $\left(i^{\text {th }}\right)$ units of the object $\left(W\left(\varepsilon\left(E_{i}\right)\right)\right)$ is the base of this inference process. For this purpose, the states of $\left(i^{\text {th }}\right)$ functional units of the object are analyzed. The inference rule to identify the state of the object is presented in the form of the following dependence:

$$
W(\varepsilon(O))=\min _{1 \leq i \leq I}\left\{W\left(\varepsilon\left(E_{i}\right)\right)\right\}
$$

where: $W(\varepsilon(O))$ - is the value of the state of the object, $W\left(\varepsilon\left(E_{i}\right)\right)$ - is the value of the state of $i^{\text {th }}$ functional unit of the object (from the set of the accepted trivalent logic of the assessment of states $-\{2,1,0\})$. 


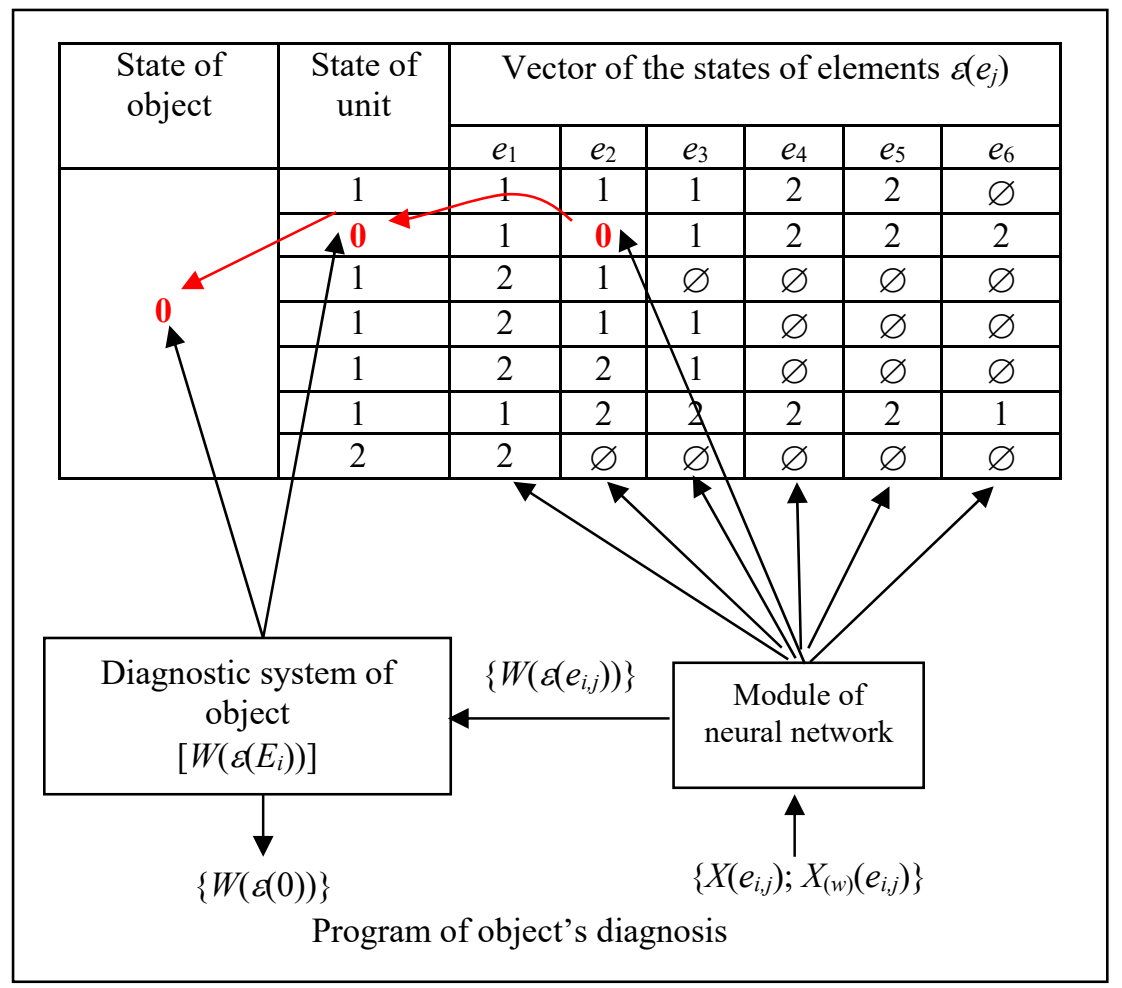

Fig. 3. Scheme of the making decisions in the diagnostic intelligent systems on the basis information from an artificial neural network.

The idea of the identification of the states of object $(W(\varepsilon(\mathrm{O})))$ on the grounds of the states having been distinguished in its structure of units $\left\{E_{i}\right\}$, which is presented in the form of dependence (4), is also graphically depicted in (Figure 3). It is evident from the analysis in (Figure 2) that if at least one of the states of units $\left(W\left(\varepsilon\left(E_{i}\right)\right)\right)$ contained in the structure of the object, is in the state $\left(W\left(\varepsilon\left(\mathrm{E}_{\mathrm{j}}\right)\right)\right)$ with the lowest valence marked from the set of states $\{2,1,0\}$, then the state of the object, which is explained by the inference rule (dependence 4 ), is such as the lowest state of this $\left(i^{\text {th }}\right)$ unit. The final result of the examination of the state of a technical object in an intelligent diagnostic system can be presented in a tabular form (cf. Table 3).

Table 3. Table of the states of object.

\begin{tabular}{|c|c|c|c|c|c|c|}
\hline $\begin{array}{c}\text { State of } \\
\text { object }\end{array}$ & \multirow{2}{*}{$\begin{array}{l}\text { State of } \\
\text { unit }\end{array}$} & \multicolumn{5}{|l|}{ Vector of the states of basic elements $\left\{\mathrm{e}_{\mathrm{i}, \mathrm{j}}\right\}$} \\
\cline { 3 - 7 } & & $\varepsilon\left(\mathrm{e}_{1,1}\right)$ & $\ldots$ & $\varepsilon\left(e_{i, j}\right)$ & $\ldots$ & $\varepsilon\left(e_{i, J}\right)$ \\
\hline \multirow{5}{*}{$W(\varepsilon(\mathrm{O})))$} & $W\left(\varepsilon\left(E_{1}\right)\right)$ & $W\left(\varepsilon\left(e_{1,1}\right)\right)$ & $\ldots$ & $W\left(\varepsilon\left(\mathrm{e}_{1, \mathrm{j}}\right)\right)$ & $\ldots$ & $W\left(\varepsilon\left(e_{I, J}\right)\right)$ \\
\cline { 2 - 7 } & $\vdots$ & $\vdots$ & $\ldots$ & $\vdots$ & $\ldots$ & $\vdots$ \\
\cline { 2 - 7 } & $W\left(\varepsilon\left(E_{i}\right)\right)$ & $W\left(\varepsilon\left(e_{i, 1}\right)\right)$ & $\ldots$ & $W\left(\varepsilon\left(e_{i, j}\right)\right)$ & $\ldots$ & $\varnothing$ \\
\cline { 2 - 7 } & $\vdots$ & $\vdots$ & $\ldots$ & $\vdots$ & $\ldots$ & $\vdots$ \\
\cline { 2 - 7 } & $W\left(\varepsilon\left(E_{I}\right)\right)$ & $W\left(\varepsilon\left(e_{I, 1}\right)\right)$ & $\ldots$ & $W\left(\varepsilon\left(e_{I, j}\right)\right)$ & $\ldots$ & $W\left(\varepsilon\left(e_{I, J}\right)\right)$ \\
\hline
\end{tabular}




\section{Conclusions}

The issues presented in the article of the creation of a set of diagnostic information concern various fields of knowledge, including technical diagnostics, the theory of operation, information technology, expert systems, fuzzy sets, artificial neural networks etc. Each of these fields is well and broadly worked out in the literature. It is the author's opinion that one can claim with a full responsibility that even the basic problem, that is the use of diagnostic information obtained in the diagnosing process of a technical object in the designing and organization of the operation process, is being constantly developed in various aspects (directions). At present, the direction of the applications of neural networks, among others in the diagnostics of technical objects, is being intensively developed.

\section{References}

1. R.E. Barlow, F. Proschan. Mathematical Theory of Reliability (J. Wiley \& Sons, New York, 1995)

2. S. Duer. Artificial neural network in the control process of object's states basis for organization of a servicing system of a technical objects. Neural Computing \& Applications 21(1), 153-160 (2012)

3. S. Duer. Examination of the reliability of a technical object after its regeneration in a maintenance system with an artificial neural network. Neural Computing \& Applications, 21(3), 523-534 (2012)

4. S. Duer, K. Zajkowski. Taking decisions in the expert intelligent system to support maintenance of a technical object on the basis information from an artificial neural network. Neural Computing \& Applications 23(7), 2185-2197 (2013)

5. S. Duer, K. Zajkowski, R. Duer, J. Paś. Designing of an effective structure of system for the maintenance of a technical object with the using information from an artificial neural network. Neural Computing \& Applications 23(3-4), 913-925 (2013)

6. S. Duer. Applications of an artificial intelligence for servicing of a technical object. Neural Computing \& Applications 22(5), 955-968 (2013)

7. M. Madan, M. Gupta, J. Liang, Homma N. Static and Dynamic Neural Networks, From Fundamentals to Advanced Theory (John Wiley \& Sons, Inc, Hoboken, New Jersey, 2003)

8. K. Zajkowski. The method of solution of equations with coefficients that contain measurement errors, using artificial neural network. Neural Computing and Applications 24(2), 431-439 (2014)

9. K. Zajkowski. An innovative hybrid insulation switch to enable/disable electrical loads without overvoltages. E3S Web of Conferences 19, 01033 (2017)

10. K. Zajkowski. Settlement of reactive power compensation in the light of white certificates. E3S Web of Conferences, 19, 01037 (2017)

11. S. Duer, K. Zajkowski, I. Płocha, R. Duer. Training of an artificial neural network in the diagnostic system of a technical object. Neural Computing \& Applications 22(7), 1581-1590 (2013)

12. K. Zajkowski, S. Scaticailov. Determination of the environmental impact of reactive power compensation in the power grid. Nonconventional Technologies Review, Romania XX(2), 54-61 (2016) 